DOI: 10.12731/2227-930X-2020-3-145-156

УДК 004.94

\title{
ПРИМЕНЕНИЕ \\ МЕТОДОВ МОДЕЛИРОВАНИЯ И ОПТИМИЗАЦИИ ИНФОРМАЦИОННЫХ СТРУКТУР ДЛЯ УПРАВЛЕНИЯ ТРАНСПОРТНЫМИ ПРЕДПРИЯТИЯМИ
}

\section{Львович Я.Е., Преображенский А.П., Чопоров О.Н.}

В данной работе рассматривается подход, связанный с управлением в компаниях, связанных с перевозками, при помощчи разных видов программного обеспечения. При решении данной задачи мь рассматривали возможности использования компьютерных технологий, методик моделирования и оптимизации.

Ключевые слова: система перевозок; алгоритм; программа; управление.

\section{APPLICATION OF METHODS OF MODELING AND OPTIMIZATION OF INFORMATION STRUCTURES FOR MANAGEMENT OF TRANSPORT ENTERPRISES}

\section{Lvovich Ya.E., Preobrazhenskiy A.P., Choporov O.N.}

In this paper, we consider an approach related to the management of transportation-related companies using various types of software. In solving this problem, we considered the possibility of using computer technology, modeling and optimization techniques.

Keywords: transportation system; algorithm; program; control.

\section{Введение}

Чтобы обеспечивать управление крупными компаниями, связанными с перевозками, в том числе и теми, которые будут территориально распределены, важно осуществлять разработку и 
использование программно-ситуационных структур. С их применением будут происходить процессы группировки по самым разным ситуациям [1]. Можно провести их объединение в множества. Ситуации будут соответствовать разным типам состояний внутри организаций. Помимо этого, для них следует, чтобы были предусмотрены соответствующие программно-целевые мероприятия. Они будут соответствовать разным направлениям функционирования транспортной компании. Важно обеспечить соответствующую поддержку по процессам, относящимся к моделированию программно-ситуационных структур.

Алгоритм моделирования использования программного обеспечения. Необходимо формировать элементы, которые будут относиться к множеству ситуаций $r=\overline{1, R}$. При этом мы применяли следующие обозначения: $R$ - будет соответствовать общему количеству таких ситуаций, которые могут наблюдаться внутри анализируемой компании, а также множеству применяемых программных типов обеспечения $v=\overline{1, V}$. Здесь мы рассматриваем обозначение $V$. Оно будет соответствовать общему числу типов программного обеспечения. В ходе моделирования такие компоненты программного обеспечения будут рассматриваться как управляющие воздействия. После того, как проведено обозначение по соответствующим множествам, необходимо, чтобы по элементам $r$ и $v$ была проведена оценка, насколько они будут соответствовать друг другу. Мы должны решать задачи управления. В этой связи в множествах элементы необходимо упорядочить. Процесс упорядочения основывается на том, что в элементах мы будем учитывать значимость в ходе принятия управленческих решений $[2,3]$. При этом необходимо ориентироваться на показатели эффективности работы транспортной компании. Отметим, как можно проводить процесс упорядочения относительно элементов, которые будут включаться в множества $R$ и $V$. Необходимо, чтобы был применен метод экспертных оценок. Вычисление экспертных оценок должно быть по нескольким направлениям. Первое связано с тем, каким образом идет контроль качества в ходе ре- 
ализации транспортных процессов. Второе связано со степенью экологичности используемых видов техники. Третье базируется на рассмотрении того, насколько эффективным образом будут использоваться разные типы ресурсов [4]. Чтобы осуществлять ход решения таких задач следует использовать метод априорного ранжирования. Затем рассмотрим особенности того, как будет применен такой метод в рассматриваемой задаче. Необходимо получать оценки по рангам $Q_{r}$ и $Q_{v}$., которые получаются при анализе множеств $R$ и $V$. В дальнейшем используется вероятностное представление для таких оценок:

$$
\begin{gathered}
p_{r}=Q_{r} / \sum_{r=1}^{R} Q_{r}, \quad r=\overline{1, R}, \quad \sum_{r=1}^{R} p_{r}=1 ; \\
p_{v}=Q_{v} / \sum_{v=1}^{V} Q_{v}, \quad v=\overline{1, V}, \quad \sum_{v=1}^{V} p_{v}=1 .(1)
\end{gathered}
$$

Для указанных выражений мы применяем следующие обозначения: $P_{r}$ - рассматривается в виде вероятностной оценки, которая демонстрирует значимость компонента $r ; P_{v}$ - рассматривается в виде вероятностной оценки, которая демонстрирует значимость компонента $v$. Для реальных ситуаций мы можем встретить разные комбинации по компонентам $r$ и $v$. Это определяется типом решаемых в компании задач, насколько они сложные [5]. В таких случаях компоненты $r$ и $v$. будут характеризоваться разной степенью неоднородности. То есть, когда происходит формирование эффективных управленческих решений, они будут иметь различную степень значимости. Проводить ее оценку можно на основе того, что рассматриваются величины энтропии

$$
H(R)=-\sum_{r=1}^{R} p_{r} \cdot \lg p_{r}, H(V)=-\sum_{v=1}^{v} p_{v} \cdot \lg p_{v} \cdot
$$

По величинам энтропии существуют соответствующие свойства, которые относятся к ее величине. Если вероятности $p_{r} p_{v}$ будут связаны с равномерным распределением, тогда величина энтропии будет расти. Чем распределение будет характеризоваться большей неравномерностью, тем по энтропии мы будем наблюдать меньшее значение. В случае максимальных значений энтропии мы можем применять такие формулы 


$$
H(R)=\lg R, H(V)=\lg V .
$$

Когда анализируются такие величины можно сделать количественное описание того, какие будут изменения в характеристиках транспортного предприятия, какие могут быть возможности его развития.

Требуется в ходе анализа проводить рассмотрение всех возможных распределений, которые характеризуют компоненты. В противном случае есть риски, связанные с тем, что для определенных комбинаций компонентов $r$ и $v$ будут учтены не все возможные варианты, относящиеся к их сочетанию. Это может быть причиной того, что управление будет совсем не эффективным. Работа системы управления должна быть одинаково, когда мы будем наблюдать разные комбинации в компонентах [6]. Поэтому уровень неоднородности будет определен рациональным способом на основе применения коэффициента неравномерности $\delta(0,4 \leq \delta \leq 0,7)$. В формуле показано, каким образом будет проведена оценка по уровню неоднородности

$$
H^{\text {pau }}(R)=\delta \cdot \lg R, H^{\text {pau }}(V)=\delta \cdot \lg V .
$$

Разные ситуации в ходе процессов перевозок описываются на базе того, что используются соответствующие элементы информационно-телекоммуникационного оборудования. Эти элементы будут оказывать разное влияние на то, как будут меняться компоненты в множестве ситуаций.

Пусть мы анализируем влияние со стороны элемента $v$ на компоненты, которые будут давать описание по множеству $r=\overline{1, R}$. Влияние может быть различным. Предлагается проводить разбиение его по диапазонам, которые будут соответствовать экспертным оценкам $Q_{r v}$, указанным в выражении

$$
Q=\left\{\begin{array}{c}
\text { существенно }(10 \text { баллов }), \\
\text { сильно }(8 \text { баллов }), \\
\text { несколько }(6 \text { баллов }), \\
\text { немного }(4 \text { балла }), \\
\text { мало }(2 \text { балла }) .
\end{array}\right.
$$

Процесс, связанный с вычислением условных вероятностей, основывается на том, что применяются значения экспертных оценок 


$$
P(r / v)=Q_{r v} / \sum_{v=1}^{V} Q_{r v}
$$

$\mathrm{C}$ тем, чтобы управление на транспортном предприятии было эффективным, важно принимать соответствующие решения. Условную энтропию мы можем анализировать в виде некоторой характеристики в ходе принятия решений. Выражения показывают, как будут вычисляться условная энтропия:

$$
H_{v}(R)=\sum_{r=1}^{R} p_{r} H_{v}(r), H_{v}(r)=-\sum_{v=1}^{V} p(r / v) \cdot \lg p(r / v) .
$$

Необходимо осуществлять совместным образом контроль качества, а также степень эффективности функционирования по всем подразделениям на предприятии, связанном с перевозками. Это показывает выражение, описывающее программно-ситуационную структуру

$$
H(R, V)=H(V)+H_{v}(R) .
$$

Как мы отмечали выше, приоритеты будут разные. Поэтому важно осуществить оценку [7] относительно адекватности модели приоритетов. Это связано с выбранным вариантом структуры

$$
H(R, V) \leq H^{\text {рац }}(R, V) .
$$

Обеспечение оптимизации процессов на транспортном предприятии. Процесс оптимизации относительно программно-ситуационной структур осуществляется на основе применения соответствующей модели. В ней дается описание приоритетов, связанных с компонентами $r$ и $v$, а также взаимодействия между ними. Поэтому требуется провести задание по альтернативным переменным [8]:

$$
x_{v}=\left\{\begin{array}{l}
1, \text { если } v-\text { е воздействие , будет включено в } \\
\text { программу работы транспорного предприятия, } v=\overline{1, V}(10) \\
0, \text { в противном случае. }
\end{array}\right.
$$

По каждому из типов программного обеспечения необходимо предусмотреть финансовые затраты. Важно учитывать, что, так как по ресурсам существуют ограничения, поэтому следует осуществлять минимизацию относительно числа элементов $v$. Важно принимать во внимание, что необходима поддержка по каждой 
ситуации $r$ со стороны мероприятий, которые будут в целевых программных компонентах. Поддержка может осуществляться не полная, но хотя бы частичным способом. Эти ограничения необходимо учитывать. При этом используем правило большинства [9]. Для этого осуществляется рассмотрение дискретной величины $C_{r v}$, которая будет характеризоваться тремя уровнями. В таких случаях вероятностные оценки $p(r / v)$ будут соотнесены с наборами значений

$$
\begin{gathered}
\mathcal{C}_{r v}=\left\{\begin{array}{c}
1, \text { если } 0,7 \leq p(r / v) \leq 1, \\
0,5, \text { если } 0,3<\mathrm{p}(\mathrm{r} / \mathrm{v})<0,7, \\
0, \text { если } 0 \leq \mathrm{p}(\mathrm{r} / \mathrm{v})<0,3 .
\end{array}\right. \\
\quad r=\overline{1, R}, v=\overline{1, V} .
\end{gathered}
$$

В указанной задаче следует учитывать две составляющих. С одной стороны, будет ограничение в ресурсах. С другой стороны, средства должны применяться целевым способом. В итоге будет управление ситуациями в процессах перевозок. Требуется при этом использовать следующую оптимизационную модель:

$$
\begin{gathered}
\sum_{v=1}^{V} z_{v} x_{v} \rightarrow \min , \\
\sum_{v=1}^{V} c_{r v} x_{v} \geq 0,5, \quad r=\overline{1, R}, \quad x_{v}=\left\{\begin{array}{l}
0 \\
1
\end{array} \quad v=\overline{1, V} .\right.
\end{gathered}
$$

Введены такие обозначения: $z_{v}$ являются прогнозируемыми затратами, которые будут связаны с тем, что реализуется $v$-й вид программного обеспечения. Происходит учет минимизации ресурсов в критерии оптимизации. При этом будут рассмотрены затраты, которые связанны с тем, что происходит реализуется программное обеспечение. Ограничения будут соответствовать не менее 50\% уровню, которые будут связаны с управленческими решениями. При этом происходит учет каждой из возможных ситуаций. Осуществлялось имитационное моделирование. В нем была применена созданная оптимизационная модель. В ходе его выполнения было рассмотрено несколько вариантов программных продуктов. Их число находится между 5 и 10. Следует использовать разные 
затраты, для того, чтобы их реализовать. При этом будет обеспечен разный уровень, на который будет происходить рост эффективности процессов, связанных с перевозками. Чтобы эксперимент был реализован, необходимо осуществить такую операцию. Альтернативные переменных $x_{v}$ будут заменены на случайные дискретные переменные $\widetilde{x}_{v}$. При этом по ним распределение будет описываться такими параметрами:

$$
p\left(\widetilde{x}_{v}=1\right)=p\left(x_{v}\right), \quad p\left(\widetilde{x}_{v}=0\right)=g_{x v}=1-p_{x v} .
$$

Была осуществлена генерация последовательности псевдослучайных чисел $v=\overline{1, V}$. Для них характерно то, что они будут иметь равномерное распределение. Такое распределение будет соответствовать интервалу $(0,1)$. Те переменные, которые будут подвергаться варьированию, должны задаваться соответствующим образом. Закономерность будет описываться при помощи такого выражения:

$$
\widetilde{x}_{v}=\left\{\begin{array}{l}
1, \text { если } v \leq p_{x v} \\
0, \text { в противном случае. }
\end{array}\right.
$$

Мы проводим рассмотрение описания имитационного эксперимента при учете следующих условий. 1. Используется вероятностный подход. 2. В оптимизационной модели происходит учет ограничений.

Анализ случайных процессов рассматривается как случайное блуждание. Описание его происходит при помощи соответствующей марковской цепи. Следует корректным образом описать состояния этой цепи. Они будут относиться к различным сочетаниям, которые будут соответствовать альтернативным переменным $x_{v}, v=\overline{1, V}$. Если мы делаем выбор сочетаний перспективных компонентов программного обеспечения, тогда их можно соотнести с поглощающими состояниями. При имитационном моделировании любой из циклов будет предоставлять возможности для того, чтобы получать перспективный вариант. Это можно объяснить особенностью марковской цепи. Вероятность перехода к поглощающему состоянию в течение определенного числа шагов будет равна единице. Переход при этом будет происходить от произвольного 
начального состояния. Для Марковской цепи есть свойство неприводимости. После осуществления имитационного моделирования, мы придем к совокупности перспективных вариантов [10]. Такие варианты рассматриваются с точки зрения экспертного анализа. Анализ проводится руководством транспортного предприятия. Тогда образуется оптимизированная совокупность программных модулей. Они будут соответствовать обозначенным целям функционирования и развития транспортного предприятия.

На рис. 1 дана схема, связанная с моделированием и оптимизацией программных средств. Она базируется на том, что применяются компьютерные технологии управления.

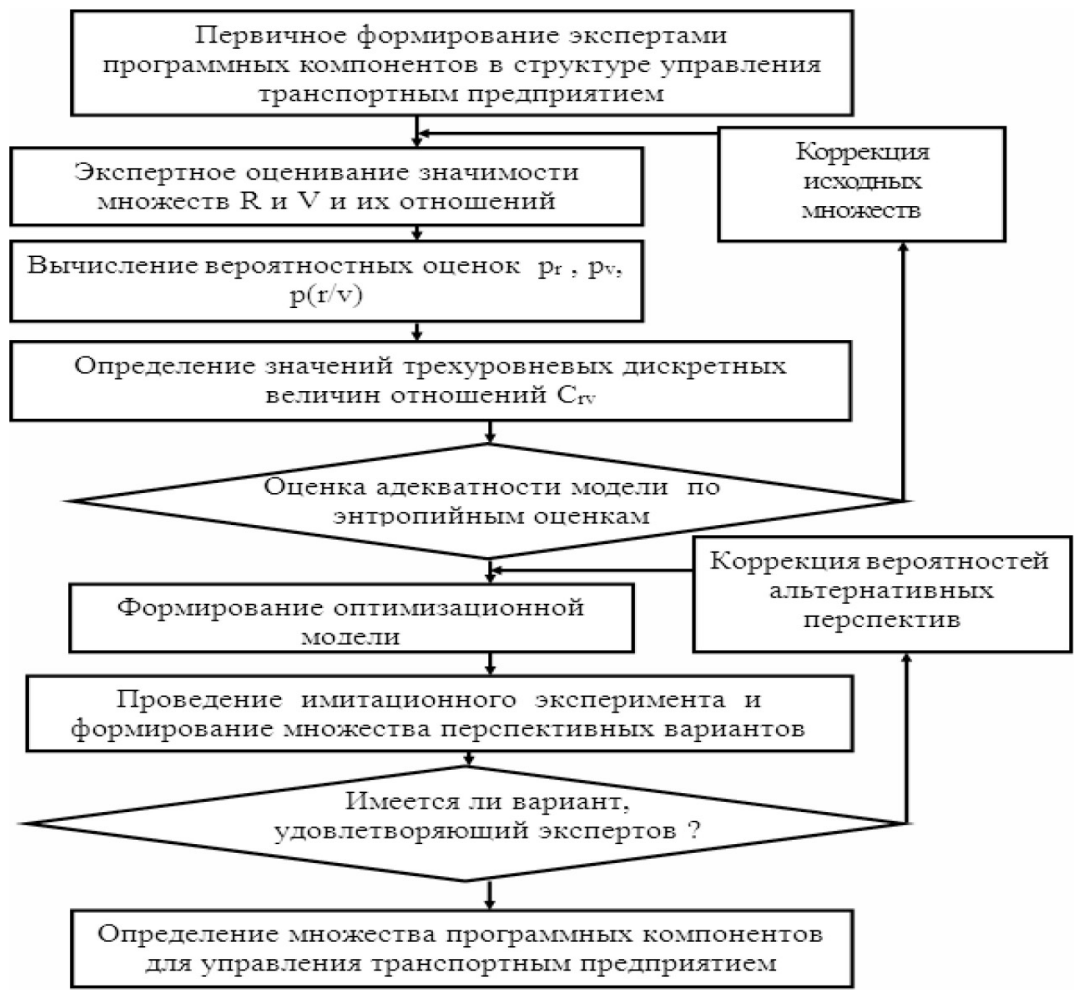

Рис. 1. Схема, показывающая процессы моделирования и оптимизации программных средств в транспортном предприятии. 


\section{Выводы}

В современных транспортных компаниях используется большое число информационно-телекоммуникационных структур. Предложен алгоритм моделирования использования программного обеспечения. Показано, как осуществляется процесс оптимизации относительно программно-ситуационной структур в транспортной компании.

Информация о конфликте интересов. Авторы заявляет об отсутствии конфликта интересов.

Информация о спонсорстве. Исследование не имело спонсорской поддержки.

\section{Список литературы}

1. Преображенский Ю.П. Информационные технологии, используемые в сфере менеджмента // Вестник Воронежского института высоких технологий. 2018. № 2 (25). С. 43-46.

2. Паневин Р.Ю., Преображенский Ю.П. Задачи оптимального управления многостадийными технологическими процессами // Вестник Воронежского института высоких технологий. 2010. № 6. С. 77-80.

3. Ермолова В.В., Преображенский Ю.П. Методика построения семантической объектной модели // Вестник Воронежского института высоких технологий. 2012. № 9. С. 87-90.

4. Преображенский Ю.П., Коновалов В.М. Анализ подходов к формированию рекомендательных систем // Вестник Воронежского института высоких технологий. 2019. № 4 (31). С. 88-90.

5. Преображенский Ю.П., Коновалов В.М. О методах создания рекомендательных систем //Вестник Воронежского института высоких технологий. 2019. № 4 (31). С. 75-79.

6. Берман Н.Д., Белов А.М. Общественный транспорт и инновации // International Journal of Advanced Studies. 2019. T. 9. № 2. С. 7-13.

7. Шакиров А.А., Зарипова Р.С. Особенности моделирования логистических систем // International Journal of Advanced Studies. 2019. T. 9. № 4. C. 27-31. 
8. Сапожникова С.М. Корпоративное управление в железнодорожном транспорте // International Journal of Advanced Studies. 2019. T. 9. № 4. C. 19-42.

9. Лысанов Д.М., Бикмухаметова Л.Т. Анализ показателей качества и конкурентоспособности оборудования // International Journal of Advanced Studies. 2018. Т. 8. № 4-2. С. 50-55.

10. Преображенский Ю.П. Построение складской структуры предприятия // Молодежь и системная модернизация страны Сборник научных статей 3-й Международной научной конференции студентов и молодых ученых. В 4-х томах. Ответственный редактор А.А. Горохов. 2018. С. 286-290.

\section{References}

1. Panevin R.Yu., Preobrazhenskij Yu.P. Zadachi optimal'nogo upravleniya mnogostadijnymi tekhnologicheskimi processami // Vestnik Voronezhskogo instituta vysokih tekhnologij. 2010. № 6. S. 77-80.

2. Preobrazhenskij Yu.P. Informacionnye tekhnologii, ispol'zuemye v sfere menedzhmenta // Vestnik Voronezhskogo instituta vysokih tekhnologij. 2018. № 2 (25). S. 43-46.

3. Ermolova V.V., Preobrazhenskij Yu.P. Metodika postroeniya semanticheskoj ob»ektnoj modeli // Vestnik Voronezhskogo instituta vysokih tekhnologij. 2012. № 9. S. 87-90.

4. Preobrazhenskij Yu.P., Konovalov V.M. Analiz podhodov k formirovaniyu rekomendatel'nyh sistem // Vestnik Voronezhskogo instituta vysokih tekhnologij. 2019. № 4 (31). S. 88-90.

5. Preobrazhenskij YU.P., Konovalov V.M. O metodah sozdaniya rekomendatel'nyh sistem //Vestnik Voronezhskogo instituta vysokih tekhnologij. 2019. № 4 (31). S. 75-79.

6. Berman N.D., Belov A.M. Obshchestvennyj transport i innovacii // International Journal of Advanced Studies. 2019. T. 9. № 2. S. 7-13.

7. Shakirov A.A., Zaripova R.S. Osobennosti modelirovaniya logisticheskih sistem // International Journal of Advanced Studies. 2019. T. 9. № 4. S. 27-31. 
8. Sapozhnikova S.M. Korporativnoe upravlenie v zheleznodorozhnom transporte // International Journal of Advanced Studies. 2019. T. 9. № 4. S. 19-42.

9. Lysanov D.M., Bikmuhametova L.T. Analiz pokazatelej kachestva i konkurentosposobnosti oborudovaniya // International Journal of Advanced Studies. 2018. T. 8. № 4-2. S. 50-55.

10. Preobrazhenskij Yu.P. Postroenie skladskoj struktury predpriyatiya // Molodezh' i sistemnaya modernizaciya strany Sbornik nauchnyh statej 3-j Mezhdunarodnoj nauchnoj konferencii studentov i molodyh uchenyh. V 4-h tomah. Otvetstvennyj redaktor A.A. Gorohov. 2018. S. 286-290.

\section{ДАННЫЕ ОБ АВТОРАХ}

Львович Яков Евсеевич, профессор, доктор технических наук Федеральное государственное образовательное учреждение выстего образования Воронежский государственный технический университет

ул. 20 лет Октября, 84, г. Воронеж, 394006, Россия Komkovvivt@yandex.ru

Преображенский Андрей Петрович, профессор, доктор технических наук, доцент Автономная некоммерческая образовательная организаџия высшего образования Воронежский институт высоких технологий ул. Ленина, 73а, Воронеж, 394043, Россия Komkovvivt@yandex.ru

Чопоров Олег Николаевич, профессор, доктор технических наук, профессор Федеральное государственное образовательное учреждение выстего образования Воронежский государственный технический университет

ул. 20 лет Октября, 84, г. Воронеж, 394006, Россия Komkovvivt@yandex.ru 


\section{DATA ABOUT THE AUTHORS}

Lvovich Yakov Yevseevich, Professor, doctor of technical Sciences, Professor

Voronezh State Technical University

84, 20 years of October Str., Voronezh, 394006, Russia

Komkovvivt@yandex.ru

ORCID: 0000-0002-7051-3763

Preobrazhenskiy Andrey Petrovich, professor, doctor of technical sciences, associate professor

Voronezh Institute of High Technologies

73a, Lenin Str., Voronezh, 394043, Russia

Komkovvivt@yandex.ru

ORCID: 0000-0002-6911-8053

Choporov Oleg Nikolaevich, Professor, doctor of technical Sciences,

Professor

Voronezh state technical University

84, 20 years of October Str., Voronezh, 394006, Russia

Komkovvivt@yandex.ru

ORCID: 0000-0002-3176-499X 